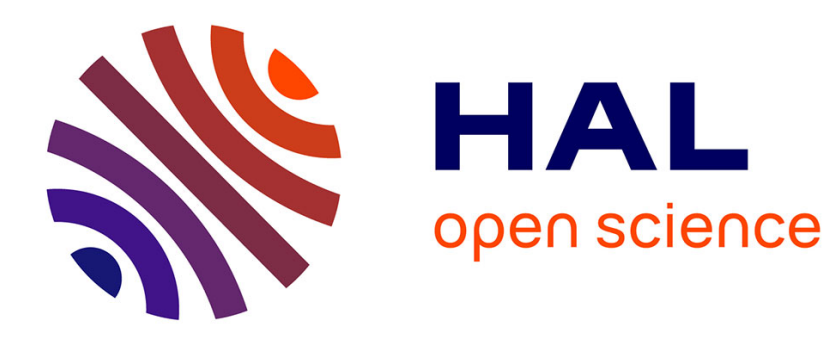

\title{
Decomposition of $S^{1}$-valued maps in Sobolev spaces
}

Petru Mironescu

\section{To cite this version:}

Petru Mironescu. Decomposition of $S^{1}$-valued maps in Sobolev spaces. Comptes rendus de l'Académie des sciences. Série I, Mathématique, 2010, 348 (13-14), pp.743-746. 10.1016/j.crma.2010.06.020 . hal-00747677

\section{HAL Id: hal-00747677 \\ https://hal.science/hal-00747677}

Submitted on 31 Oct 2012

HAL is a multi-disciplinary open access archive for the deposit and dissemination of scientific research documents, whether they are published or not. The documents may come from teaching and research institutions in France or abroad, or from public or private research centers.
L'archive ouverte pluridisciplinaire HAL, est destinée au dépôt et à la diffusion de documents scientifiques de niveau recherche, publiés ou non, émanant des établissements d'enseignement et de recherche français ou étrangers, des laboratoires publics ou privés. 


\title{
Decomposition of $\mathbb{S}^{1}$-valued maps in Sobolev spaces
}

\author{
Petru Mironescu *
}

June 22nd, 2010

\begin{abstract}
Let $n \geq 2, s>0, p \geq 1$ be such that $1 \leq s p<2$. We prove that for each map $u \in W^{s, p}\left(\mathbb{S}^{n} ; \mathbb{S}^{1}\right)$ one can find $\varphi \in W^{s, p}\left(\mathbb{S}^{n} ; \mathbb{R}\right)$ and $v \in W^{s p, 1}\left(\mathbb{S}^{n} ; \mathbb{S}^{1}\right)$ such that $u=v e^{\imath \varphi}$. This yields a decomposition of $u$ into a part that has a lifting in $W^{s, p}, e^{\imath \varphi}$, and a map "smoother" than $u$ but without lifting, namely $v$. Our result generalizes a previous one of Bourgain and Brezis (which corresponds to the case $s=1 / 2, p=2$ ). As a consequence, we find an intuitive proof for the existence of the distributional Jacobian $J u$ of maps $u \in W^{s, p}\left(\mathbb{S}^{n} ; \mathbb{S}^{1}\right)$ (originally due to Bourgain, Brezis and the author). By completing a result of Bousquet, we characterize the distributions of the form $J u$.

\section{Résumé}

Décomposition des applications unimodulaires dans les espaces de Sobolev. Soient $n \geq 2, s>0, p \geq 1$ tels que $1 \leq s p<2$. Nous montrons que, pour chaque $u \in W^{s, p}\left(\mathbb{S}^{n} ; \mathbb{S}^{1}\right)$, il existe $\varphi \in W^{s, p}\left(\mathbb{S}^{n} ; \mathbb{R}\right)$ et $v \in W^{s p, 1}\left(\mathbb{S}^{n} ; \mathbb{S}^{1}\right)$ tels que $u=v e^{\imath \varphi}$. Ceci donne une décomposition de $u$ comme produit d'un facteur qui se relève dans $W^{s, p}, e^{\imath \varphi}$, et d'un facteur "plus régulier" que $u$ mais qui ne se relève pas, à savoir $v$. Notre décomposition généralise un résultat antérieur de Bourgain et Brezis (qui ont traité le cas $s=1 / 2, p=2$ ). Une conséquence de notre résultat est une preuve intuitive de l'existence du jacobien au sens des distributions $J u$ pour les applications $u \in W^{s, p}\left(\mathbb{S}^{n} ; \mathbb{S}^{1}\right)$ (résultat dû, avec un argument différent, à Bourgain, Brezis et l'auteur). En complétant un résultat de Bousquet, nous caractérisons les distributions de la forme $J u$.
\end{abstract}

\section{Decomposition of $\mathbb{S}^{1}$-valued maps}

Our main result is the following

Theorem 1 Let $n \geq 2, s>0, p \geq 1$ be such that $1 \leq s p<2$. Let $u \in W^{s, p}\left(\mathbb{S}^{n} ; \mathbb{S}^{1}\right)$. Then there exist $\varphi \in W^{s, p}\left(\mathbb{S}^{n} ; \mathbb{R}\right)$ and $v \in W^{s p, 1}\left(\mathbb{S}^{n} ; \mathbb{S}^{1}\right)$ such that $u=v e^{\imath \varphi}$.

In addition, we have (with $|\cdot|_{W^{r, q}}$ standing for the semi-norm given by the highest order term in $\left.\|\cdot\|_{W^{r, q}}\right)$

$$
|\varphi|_{W^{s, p}} \lesssim|u|_{W^{s, p}},|v|_{W^{s p, 1}} \lesssim|u|_{W^{s, p}}^{p}
$$

The special case $s=1 / 2, p=2$ of Theorem 1 is due to Bourgain and Brezis [4]. (In [4], $u$ is supposed to be in the $H^{1 / 2}$-closure of $C^{\infty}\left(\mathbb{S}^{n} ; \mathbb{S}^{1}\right)$. This extra assumption was removed in [6].) In Theorem 1 , $\mathbb{S}^{n}$ does not play special role; one could replace, e. g., $\mathbb{S}^{n}$ by any smooth bounded simply connected domain. Theorem 1 yields a satisfactory substitute to the lifting theory in $W^{s, p}\left(\mathbb{S}^{n} ; \mathbb{S}^{1}\right)$, theory

${ }^{*}$ Université de Lyon ; CNRS ; Université Lyon 1 ; Institut Camille Jordan, 43 blvd du 11 novembre 1918, F-69622 Villeurbanne-Cedex, France. Email address: mironescu@math.univ-lyon1.fr 
developed successively in [5], [18] and [14]. As proved in these papers, when $n \geq 2$ and $s p \notin[1,2)$, one may characterize maps $u \in W^{s, p}\left(\mathbb{S}^{n} ; \mathbb{S}^{1}\right)$ in terms of their liftings. (For a precise statement, we refer to [15], Theorem 6.1, p. 15.) However, when $1 \leq s p<2$, there is no satisfactory description of maps in terms of their phases. A typical example is the map $\mathbb{C} \ni z \mapsto z /|z|$, which belongs to $W^{s, p}(B(0,1))$ when $s p<2$, but does not have a phase better than $z \mapsto \arg z$, which merely belongs to BV. Our result allows to decompose $u$ into two parts, one as smooth as $u$ and which admits a lifting in $W^{s, p}$, the other one without lifting in $W^{s, p}$, but "smoother" than $u$. In Theorem 1, one cannot replace $W^{s, p}$ (for $\varphi$ ) or $W^{s p, 1}$ (for $v$ ) by smaller Sobolev spaces.

The proof of Theorem 1 is constructive: there is an explicit formula giving $\varphi$. Part of the proof is inspired by similar constructions of Bourgain and Brezis [4 and of the author [14]. We describe the main lines of the proof when $s<1$ and $1 \leq s p<2$, and when $\mathbb{S}^{n}$ is replaced by $B$, the unit ball in $\mathbb{R}^{n}$. We extend $u \in W^{s, p}\left(B ; \mathbb{S}^{1}\right)$ to $\mathbb{R}^{n}$ by reflections and cutoff. We let $\Pi \in C^{\infty}\left(\mathbb{R}^{2} ; \mathbb{R}^{2}\right)$ such that $\Pi(z)=z /|z|$ when $|z| \geq 1 / 2$ and let $\rho$ be a suitable mollifier. With $w(x, \varepsilon):=u * \rho_{\varepsilon}(x), x \in \mathbb{R}^{n}, \varepsilon>0$, we set, inspired by [14],

$$
\varphi_{1}(x):=-\int_{0}^{\infty} \Pi \circ w(x, \varepsilon) \wedge \frac{\partial}{\partial \varepsilon}(\Pi \circ w)(x, \varepsilon) d \varepsilon .
$$

This $\varphi_{1}$ satisfies $\varphi_{1} \in W^{s, p}(B) U:=u e^{-\imath \varphi_{1}} \in W^{1, s p}(B)$. If $s p=1$, then we may take $\varphi=\varphi_{1}$. When $1<s p<2$, two more steps are needed. We extend $U$ to $\mathbb{R}^{n}$ by reflections and cutoff and define

$\varphi_{2}:=\sum_{k} \sum_{j<k} U_{j} \wedge U_{k}$. Here, $U=\sum U_{j}$ is a Littlewood-Paley decomposition of $U$. The idea of improving the regularity of a map with the help of this phase originates in the paper [4] of Bourgain and Brezis. This $\varphi_{2}$ satisfies $\varphi_{2} \in W^{1, s p}$ and $U e^{-\imath \varphi_{2}} \in W^{s p, 1}(B)$.

Third step: since $\varphi_{2} \in W^{1, s p}$, we have $\varphi_{2}=\varphi_{3}+\varphi_{4}$, where $\varphi_{3} \in W^{s, p}$ and $\varphi_{4} \in W^{s p, 1} \cap W^{1, s p}$. The regularity of $\varphi_{4}$ implies that $e^{\imath \varphi_{4}} \in W^{s p, 1}$ [10], [13]. Thus $u=e^{\imath \varphi} v$, where $\varphi:=\varphi_{1}+\varphi_{3} \in W^{s, p}$ and $v:=U e^{\imath \varphi_{4}} \in W^{s p, 1}$.

\section{The distributional Jacobian revisited}

We recall the definition of the distributional Jacobian for $\mathbb{S}^{1}$-valued maps [17, [19, [2], [9], [12], [1], [6], [7]. If $u=\left(u_{1}, u_{2}\right) \in W^{1,1}\left(\mathbb{S}^{2} ; \mathbb{S}^{1}\right)$, then $J u:=\frac{1}{2} d\left(u_{1} d u_{2}-u_{2} d u_{1}\right)$. This distribution (current) coincides with the usual Jacobian 2-form $d u_{1} \wedge d u_{2}$ if $u$ is sufficiently smooth, say $u \in H^{1}$. In the latter case, $J u=0$ for $\mathbb{S}^{1}$-valued maps $u$. As a distribution, $J u$ is defined by

$$
\langle J u, \zeta\rangle=\frac{1}{2} \int_{\mathbb{S}^{2}}\left(u_{1} d u_{2}-u_{2} d u_{1}\right) \wedge d \zeta, \quad \forall \zeta \in C^{\infty}\left(\mathbb{S}^{2} ; \mathbb{R}\right) .
$$

More generally, when $u \in W^{1,1}\left(\mathbb{S}^{n} ; \mathbb{S}^{1}\right), J u$ is defined as an $(n-2)$-current through the formula

$$
\langle J u, \zeta\rangle=\frac{1}{2} \int_{\mathbb{S}^{n}}\left(u_{1} d u_{2}-u_{2} d u_{1}\right) \wedge d \zeta, \quad \forall \zeta \in \Lambda^{n-2}\left(\mathbb{S}^{n}\right) .
$$

The following result was proved in [6].

Theorem 2 ([6]) Let $n \geq 2, s>0, p \geq 1$ be such that $1 \leq s p<2$. Then $W^{s, p} \cap W^{1,1}\left(\mathbb{S}^{n} ; \mathbb{S}^{1}\right)$ is dense in $W^{s, p}\left(\mathbb{S}^{n} ; \mathbb{S}^{1}\right)$. In addition, the map $u \mapsto J u$ extends by continuity from $W^{s, p} \cap W^{1,1}\left(\mathbb{S}^{n} ; \mathbb{S}^{1}\right)$ to $W^{s, p}\left(\mathbb{S}^{n} ; \mathbb{S}^{1}\right)$.

Denoting by $u \mapsto J u$ this extension, Theorem 1 sheds a new light on Theorem 2 via the following 
Proposition 3 Let $n \geq 2, s>0, p \geq 1$ be such that $1 \leq s p<2$. Let $u \in W^{s, p}\left(\mathbb{S}^{n} ; \mathbb{S}^{1}\right)$ and write $u=v e^{\imath \varphi}$, with $\varphi \in W^{s, p}$ and $v \in W^{s p, 1}$. Then, for each choice of $\varphi$ and $v$, we have

$$
\langle J u, \zeta\rangle=\frac{1}{2} \int_{\mathbb{S}^{n}}\left(v_{1} d v_{2}-v_{2} d v_{1}\right) \wedge d \zeta, \quad \forall \zeta \in \Lambda^{n-2}\left(\mathbb{S}^{n}\right) .
$$

\section{Existence of maps with prescribed singularities. The two dimensional case}

Set $\mathcal{R}:=\left\{u \in W^{1,1}\left(\mathbb{S}^{2} ; \mathbb{S}^{1}\right) ; u\right.$ is smooth outside some finite set $\left.A=A(u)\right\}$. When $u \in \mathcal{R}$, we have $\langle J u, \zeta\rangle=\pi \sum_{a \in A} d_{a} \zeta(a)$, where the integers $d_{a}$ are the degrees of $u$ on suitably oriented small circles around $a \in A$ and satisfy $\sum d_{a}=0$ [12]. Thus $J u=\pi \sum_{a \in A} d_{a} \delta_{a}$. Since $\mathcal{R}$ is dense in $W^{1,1}\left(\mathbb{S}^{2} ; \mathbb{S}^{1}\right)[3$, one obtains that $\left\{J u ; u \in W^{1,1}\left(\mathbb{S}^{2} ; \mathbb{S}^{1}\right)\right\} \subset E_{1,1}$, where $E_{1,1}:=\pi \overline{\left\{\sum\left(\delta_{P_{j}}-\delta_{N_{j}}\right)\right\}}{ }^{\left(W^{1, \infty}\right)^{*}}$. The reversed inclusion is true.

Theorem 4 ([1], [1] $)$ We have $\left\{J u ; u \in W^{1,1}\left(\mathbb{S}^{2} ; \mathbb{S}^{1}\right)\right\}=E_{1,1}$.

Bousquet [7] partially completed this result.

Theorem 5 ([7]) Assume that $s \geq 1$ and $1 \leq s p<2$. Then $\left\{J u ; u \in W^{s, p}\left(\mathbb{S}^{2} ; \mathbb{S}^{1}\right)\right\}=E_{s, p}$, where

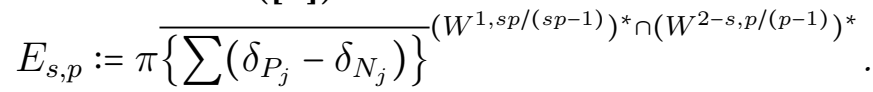

Note that the definition of $E_{s, p}$ suggests that different values of $s$ and $p$ yield different $E_{s, p}$ 's. Our first result in this direction is somewhat surprising.

Theorem 6 Assume that $s \geq 1$ and $1 \leq s p<2$. Then $E_{s, p}=E_{1, s p}$. In particular, if a (possible infinite) sum of the form $\sum\left(\delta_{P_{j}}-\delta_{N_{j}}\right)$, with $\sum\left|P_{j}-N_{j}\right|<\infty$, acts on $W^{1, r}$ for some $r \in(2, \infty)$, then it also acts on the Hölder space $C^{2-r /(r-1)}$.

As a byproduct, the proof of the above theorem yields the following curious estimate

$$
\left\|\sum\left(\delta_{P_{j}}-\delta_{N_{j}}\right)\right\|_{\left(C^{\alpha}\right)^{*}} \leq K_{\alpha}\left\|\sum\left(\delta_{P_{j}}-\delta_{N_{j}}\right)\right\|_{\left(W^{1,(2-\alpha) /(1-\alpha))^{*}}\right.}^{2-\alpha},
$$

with $K_{\alpha}$ depending on $0<\alpha<1$ but independent of the $P_{j}$ 's and $N_{j}$ 's.

Our next result completes Theorem 5 .

Theorem 7 Assume that $1 \leq s p<2$. Then $\left\{J u ; u \in W^{s, p}\left(\mathbb{S}^{2} ; \mathbb{S}^{1}\right)\right\}=E_{1, s p}$.

\section{Existence of maps with prescribed singularities. The higher dimensional case}

In dimension 3 or higher, the class $\mathcal{R}$ is defined as

$\mathcal{R}:=\left\{u \in W^{1,1}\left(\mathbb{S}^{n} ; \mathbb{S}^{1}\right) ; u\right.$ is smooth outside some $(n-2)$-submanifold without boundary $A=A(u)$ of $\left.\mathbb{S}^{n}\right\}$.

If $u \in \mathcal{R}$, then we may identify $J u$ with the $(n-2)$-current $\pi \sum d_{j} \int_{\Gamma_{j}}$, where $\Gamma_{j}$ are the (orientable, without boundary) connected components of $A$ and the integers $d_{j}$ are the degrees of $u$ 
on suitably oriented small circles linking to the $\Gamma_{j}$ 's [12], [1], [7]. We then define, for $1<q<2$,

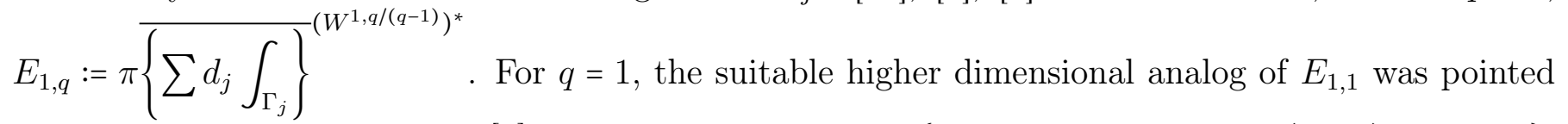
out by Alberti, Baldo, Orlandi [1] and is given by $E_{1,1}:=\pi\{\partial M ; M$ is a rectifiable $(n-1)-$ current $\}$. With these notations, we have

Theorem 8 Assume that $n \geq 3$ and $1 \leq s p<2$. Then $\left\{J u ; u \in W^{s, p}\left(\mathbb{S}^{n} ; \mathbb{S}^{1}\right)\right\}=E_{1, s p}$.

The case $s=1, p=1$ was known before [1]. The case $s p=1$ was obtained jointly with Bousquet [8]. The case $1<s p<2$ relies on Theorem 1 and on techniques from [7]. Finally, the analog of (5) is given by

$$
\left\|\sum d_{j} \int_{\Gamma_{j}}\right\|_{\left(C^{\alpha}\right)^{*}} \leq K_{\alpha}\left\|\sum d_{j} \int_{\Gamma_{j}}\right\|_{\left(W^{1,(2-\alpha) /(1-\alpha)}\right)^{*}}^{2-\alpha}, 0<\alpha<1 .
$$

Detailed proofs will appear in [16].

\section{Acknowledgement}

The author warmly thanks Pierre Bousquet and Haïm Brezis for useful discussions.

\section{References}

[1] G. Alberti, S. Baldo, G. Orlandi, Functions with prescribed singularities, J. Eur. Math. Soc. 5 (2003), no. 3, 275-311.

[2] J. M. Ball, Convexity conditions and existence theorems in nonlinear elasticity, Arch. Ration. Mech. Anal. 63 (1977), 337-403.

[3] F. Bethuel, X. Zheng, Density of Smooth Functions between Two Manifolds in Sobolev Spaces, J. Funct. Anal. 80 (1988), 60-75.

[4] J. Bourgain, H. Brezis, On the equation $\operatorname{div} Y=f$ and application to control of phases, J. Amer. Math. Soc. 16 (2003), 393-426.

[5] J. Bourgain, H. Brezis, P. Mironescu, Lifting in Sobolev spaces, J. Anal. Math. 80 (2000), 37-86.

[6] J. Bourgain, H. Brezis, P. Mironescu, $H^{1 / 2}$ maps with values into the circle: minimal connections, lifting, and the Ginzburg-Landau equation, Publ. Math. Inst. Hautes Études Sci. 99 (2004), 1115.

[7] P. Bousquet, Topological singularities in $W^{s, p}\left(\mathbb{S}^{N}, \mathbb{S}^{1}\right)$, Journal d'Analyse Mathématique 102 (2007), 311-346.

[8] P. Bousquet, P. Mironescu, in preparation.

[9] H. Brezis, J.-M. Coron, E. Lieb, Harmonic maps with defects, Comm. Math. Phys. 107 (1986), no. 4, 649-705.

[10] H. Brezis, P. Mironescu, Gagliardo-Nirenberg, composition and products in fractional Sobolev spaces, J. Evol. Equ. 1 (2001), 387-404.

[11] H. Brezis, P. Mironescu, A. Ponce, $W^{1,1}$-maps with values into $\mathbb{S}^{1}$, in Geometric analysis of PDE and several complex variables (S. Chanillo. P. D. Cordaro, N. Hanges, J. Hounie, A. Meziani eds), Contemporary Mathematics, Amer. Math. Soc., Providence, RI, 368 (2005), 69-100. 
[12] R. L. Jerrard, H. M. Soner, Functions of bounded higher variation, Indiana Univ. Math. J. 51 (2002), 645-677.

[13] V. Maz'ya, T. Shaposhnikova, An elementary proof of the Brezis and Mironescu theorem on the composition operator in fractional Sobolev spaces, J. Evol. Equ. 2 (2002), no. 1, 113-125.

[14] P. Mironescu, Lifting default for $\mathbb{S}^{1}$-valued maps, C. R. Acad. Sci. Paris, Ser. I 346 (2008), nos. 19-20, 1039-1044.

[15] P. Mironescu, $\mathbb{S}^{1}$-valued Sobolev maps, http://math.univ-lyon1.fr/ ${ }^{\text {mironescu/7.pdf }}$

[16] P. Mironescu, Sobolev spaces of circle-valued maps, in preparation.

[17] C. B. Morrey, Multiple integrals in the calculus of variations, Die Grundlehren der mathematischen Wissenschaften, vol. 130, Springer-Verlag New York, Inc., New York, 1966.

[18] H.-M. Nguyen, Inequalities related to liftings and applications, C. R. Acad. Sci. Paris, Ser. I 346 (2008), nos. 17-18, 957-962.

[19] Y. G. Reshetnyak, The weak convergence of completely additive vector-valued set functions, Sibirsk. Mat. Z. 9 (1968), 1386-1394. 